\title{
Hypermethylation of TET1 Promoter Is a New Diagnosic Marker for Breast Cancer Metastasis
}

\author{
Yi Sang ${ }^{1}$, Chun Cheng ${ }^{1}$, Xiao-Feng Tang ${ }^{1}$, Mei-Fang Zhang ${ }^{2}$, Xiao-Bin Lv ${ }^{1 *}$
}

\begin{abstract}
Breast cancer metastasis is a major cause of cancer-related death in women. However, markers for diagnosis of breast cancer metastasis are rare. Here, we reported that TET1, a tumor suppressor gene, was downregulated and hypermethylated in highly metastatic breast cancer cell lines. Moreover, silencing of TET1 in breast cancer cells increased the migration and spreading of breast cancer cells. In breast cancer clinical samples, TET1 expression was reduced in LN metastases compared with primary tissues. Besides, the methylation level of the TET1 promoter was increased significantly in LN metastases. Taken together, these findings indicate that promoter hypermethylation may contribute to the downregulation of TET1 and could be used as a promising marker for diagnosis in patients with breast cancer metastasis.
\end{abstract}

Keywords: TET1 - breast cancer metastasis - methylation

Asian Pac J Cancer Prev, 16 (3), 1197-1200

\section{Introduction}

Breast cancer metastasis is a major cause for the failure of treatment in women, making it crucial to identify proteins and signaling pathways involved in tumor cell dissemination(Hong et al., 2012; Truong et al., 2014). Also, metastasis is a multistep process involving cell detachment from the primary tumor, extravasation, and invasion to the secondary organ(Lv et al., 2011; Li et al., 2014). During the metastasis process, the oncogenes are activated and the tumor suppressor genes are inactivated, which may be responsible for breast cancer metastasis (Hong et al., 2012; Liu et al., 2013; Cao et al., 2014). Promoter hypermethylation of tumor suppressor genes is one of the ways to suppress its expression during the breast cancer metastasis (Lv et al., 2011).

The TET (ten-eleven translocation) family proteins are dioxygenases that utilize two key co-factors: Fe(II) and 2-oxoglutrate(2OG), to convert successively the methyla group of 5-methylcytosine (5-mC) to hydroxymethyl ,formyl, or carboxyl groups, thus forming the oxidized methycytosines 5-hydroxymethylcytosine (5-hmC), 5-formylcytosine (5-fC), and 5-carboxycytosine (5-caC) (Tahiliani et al., 2009; He et al., 2011; Gan et al., 2013; Pastor et al., 2013; Wu and Zhang, 2014). A subsequent decarboxylation of 5-caC, by etiher a theymine-DNA glycosylase or other DNA repair enzymes, could then lead to eventual DNA demethylation and promote genes expression (Yang et al., 2013; Mariani et al., 2014). There are three TET genes in mammalian cells: TET1, TET2 and TET3(Pastor et al., 2013). Furthermore, deregulation of the three proteins were accompanied by some diseases such as cancers(Kudo et al., 2012; Lian et al., 2012). TET1 act as tumor suppressors regulating cancer development, growth and invasion (Mercher et al., 2012; Ciccarone et al., 2014). However, the mechanism of TET1 downregulation in tumors are not clearly addressed.

Here, we report that TET1 expression was reduced in metastatic breast cancer cells and tumor tissues and inversely correlated with the methylation level of TET1 promoter. In addition, modulation of TET1 level by siRNAs further increased the migratory ability of breast cancer cells. Our results may offer a new strategy for diagnosis and therapy of breast cancer metastasis.

\section{Materials and Methods}

\section{Cell culture and samples collection}

Human breast cancer cell lines (BT474, SK-BR3, BT549, MDA-MB-231) were cultured in Dulbecco's modified Eagle's medium (DMEM, Life) supplemented with $10 \%$ fetal bovine serum (HyClone). All cell lines were incubated in a humidified chamber with $5 \% \mathrm{CO} 2$ at $37^{\circ} \mathrm{C}$. Twenty-eight fresh primary breast cancer primary tissues and Twenty-eight fresh breast cancer with lymph node metastasis were obtained at the time of diagnosis before any therapy from Sun Yat-sen University Cancer Center (Guangzhou, China). The clinical processes were approved from the Ethics Committees of Sun Yat-sen University and the informed consent was collected from each patient.

${ }^{I}$ Department of Center Laboratory, the Third Affiliated Hospital, Nanchang University, Nanchang, ${ }^{2}$ State Key Laboratory of Oncology in South China, Sun Yat-Sen University Cancer Center, Guangzhou, China ${ }^{\&}$ Equal contributors *For correspondence: promab2006@126.com 
RNA extraction and quantitative real-time PCR ( $q R T$ $P C R)$

qRT-PCR was performed as described previously(Gong et al., 2014). Total RNA of tissue specimens was isolated using the AllPrep DNA/RNA Mini Kit (Qiagen, "80204, Germany) according to the manufacturer's instructions. First-strand cDNA was synthesized using PrimeScript ${ }^{\circledR}$ RT reagent Kit (Takara, Japan) with gDNA Eraser. Quantitative PCR was performed for detection of TET1 mRNA using SYBR ${ }^{\circledR}$ Premix Ex Taq ${ }^{\mathrm{TM}}$ II (Takara,Japan). The sequences of primers were as follows: for TET1: 5' CTTGCCTGGACTTCTGTGC 3' (forward), 5' AAAGAGCGGTTATCTTCTCG 3' (reverse); for GAPDH: 5'ACAGTCAGCCGCATCTTCTT3' (forward), 5' GACAAGCTTCCCGTTCTCAG3' (reverse). The PCR condition was: $95^{\circ} \mathrm{C}$ for 4 minutes, followed by 40 cycles of $95^{\circ} \mathrm{C}$ for $20 \mathrm{~s}, 60^{\circ} \mathrm{C}$ for 20 and $70^{\circ} \mathrm{C}$ for $30 \mathrm{~s}$.

\section{Oligonucleotide transfection}

Small interfering RNAs (siRNAs) specifically targeting human TET1 were designed according to previously validated oligonucleotides(Hsu et al., 2012): 5'-CCCAGAAGATT TAGAATTGAT-3' and were synthesized by GenePharma (Shanghai, China). siRNA against GFP (si-GFP) bought from GenePharma (Shanghai, China) were used as the negative control. The siRNAs were transfected into cells at a working concentration of $50 \mathrm{nmol} / \mathrm{L}$ with the RNAiMAX reagent (Life, USA) according to the manufacturer's instructions.

\section{Wound-healing assay}

Cell motility was assessed by measuring the movement of cells into a scraped, acellular area created by a 200 $\mu$ pipette tube, and the spread of wound closure was observed after $24 \mathrm{~h}$ and $48 \mathrm{~h}$ and photographed under a microscope.

\section{Transwell assay}

For the transwell invasion assay, $3.5 \times 10^{5}$ cells in $200 \mu \mathrm{l}$ of serum-free DMEM were added to the cell culture inserts with an 8- $\mu \mathrm{m}$ microporous filter with extracellular matrix coating (Becton Dickinson Labware, Bedford ,MA). The DMEM medium containing 10\% FBS was added to the bottom chamber. After 24 hours of incubation, the cells in the lower surface of the filter were fixed and stained followed by microscopic examination. The number of migrated cells in three random optical fields $(\times 100$ magnification) from triplicate filters was averaged.

\section{Genomic DNA isolation and bisulfite DNA sequencing PCR (BSP) analysis}

BSP assays were performed as described previously $(\mathrm{Lv}$ et al., 2011). Briefly, genomic DNA was extracted from breast cancer cell lines or tissues using the AllPrep DNA/ RNA Mini Kit (Qiagen, "80204, Germany). A total of $1 \mu \mathrm{g}$ of DNA was treated with sodium bisulfite using a Zymo DNA Modication Kit (Zymo Research, Hornby, Canada). Bisulfite DNA sequencing was performed to assess the methylation levels of twenty-two $\mathrm{CpG}$ sites spanning +202 to +534 of variant (NM_030625.2) within the TET1 promoter. Nucleotide sequences of the primers used in bisulfate genomic sequencing were as follows: F: 5'-GGGTTTTGATTGTGTTGG -3', R: 5'ACCCACCCCTAAAACAAC -3'.

\section{Cell spreading assay}

Cells were lifted by trypsinization, pelleted, resuspended in serum-free media and incubated at 37 ${ }^{\circ} \mathrm{C}$ for $1 \mathrm{~h}$ with gentle agitation. The cells were pelleted, resuspended in media with $0.2 \% \mathrm{FBS}$, and then allowed to spread on the matrigel-coated plate. After $2.5 \mathrm{~h}$, cells were fixed with $3.7 \%$ formaldehyde and photographed. Spread cells were defined as cells with irregular morphology and lacking phase brightness; non-spread cells were rounded and phase-bright under the microscope. Multiple fields were imaged and 200 transfected cells were monitored and counted blindly for each experiment.

\section{Results}

TET1 expression was down-regulated and inversely correlated with its promoter hypermethylation level in metastatic breast cancer cell lines

TET1 was identified as a tumor suppressor gene and down-regulated TET1 predicted a poor outcome in breast cancer (Hsu et al., 2012; Sun et al., 2013). However, the expression level of TET1 is unclear during breast cancer metastasis. To address whether TET1 expression was attenuated in metastatic breast cancer cell lines, we detected the mRNA level of TET1 in a panel of breast cancer cell lines with different metastatic capacity. As shown in figure 1A, the mRNA level of TET1 was significantly down-regulated in metastatic breast cancer
A

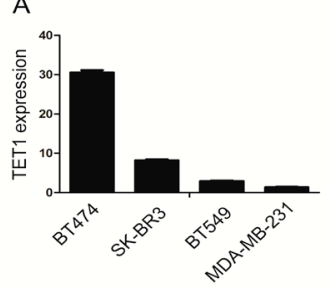

C

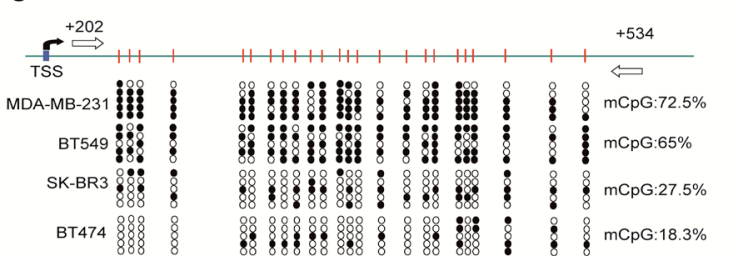

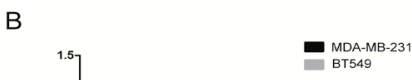

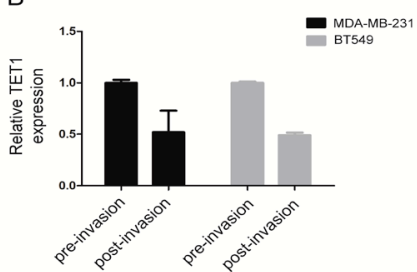

Figure 1. The Hypermethylation of TET1 Promoter in Highly Metastatic Breast Cancer Cells and Caused it to Express Low Level. A. The mRNA levels of TET1 were determined in four breast cancer cell lines by qRT-PCR. GAPDH was used as the internal control. B. The mRNA levels of TET1 were determined in the transwell pre-invasion and post-invasion in MDA-MB-231 and BT549, respectively. GAPDH was used as the internal control. C. The TET1 promoter was amplified from bisulfite-converted DNA and cloned into a pMD18T TAcloning vector. The status of the methylated cytosine residues within the TET1 promoter was evaluated by sequencing 5 colonies randomly picked in the indicated breast cancer cell lines. Each red bar represents a CpG site. The open ovals indicate unmethylated $\mathrm{CpG}$ sites and the solid ovals indicate methylated sites 
TET1 Promoter Hypermethylation Is a New Diagnosic Marker for Breast Cancer Metastasis

cells, MDA-MB-231 and BT549, compared to nonmetastatic BT474 and SK-BR3 cells. To further confirm this finding, a cell invasion model was used to detect TET1 expression, as shown in Figure 1B, the expression of TET1 decreased in highly invasive cells than the parental cells in MDA-MB-231.

To investigate the potential mechanism by which TET1 is down-regulated in metastatic breast cancer cells, we analyzed the promoter sequence of TET1 and found a $\mathrm{CpG}$ island located around the transcriptional start site of TET1(Figure 1C). Bisulfite sequencing PCR (BSP) was used to detect the methylation status of breast cancer cell lines with different metastatic capacity. As shown in figure $1 \mathrm{C}$, the $\mathrm{CpG}$ island of TET1 promter was heavily methylated in aggressive MDA-MB-231 and BT549 cells but only methylated at low levels in nonmetastatic BT474 and SK-BR3 cells. These results indicate that the mRNA level of TET1 is reversely correlate with its promoter methylation level in breast cancer cells.

\section{TET1 inhibits the invasion of breast cancer cells}

The above results led us to investigate the role of TET1 during breast cancer metastasis. BT474 cells was transiently transfected with TET1 siRNAs and the silence of TET1 was evaluated by qRT-PCR (Figure 2A). Notably, we observed major increased migratory ability of BT474 cells transfected with TET1 siRNA compared to control cells with both transwell assay and wound healing assay (Figure 2B and 2C). The phenotype of the TET1 deletion is consistent with that observed in a previous study which using the Real-Time Cell Analyzer (RTCA) Dual Plate (DP) system(Hsu et al., 2012). To determine whether TET1 may influence cell spreading in breast cancer, BT474 cells were transfected with siRNA or negative

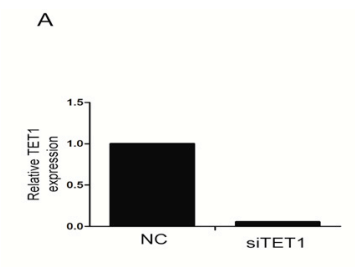

$\mathrm{C}$
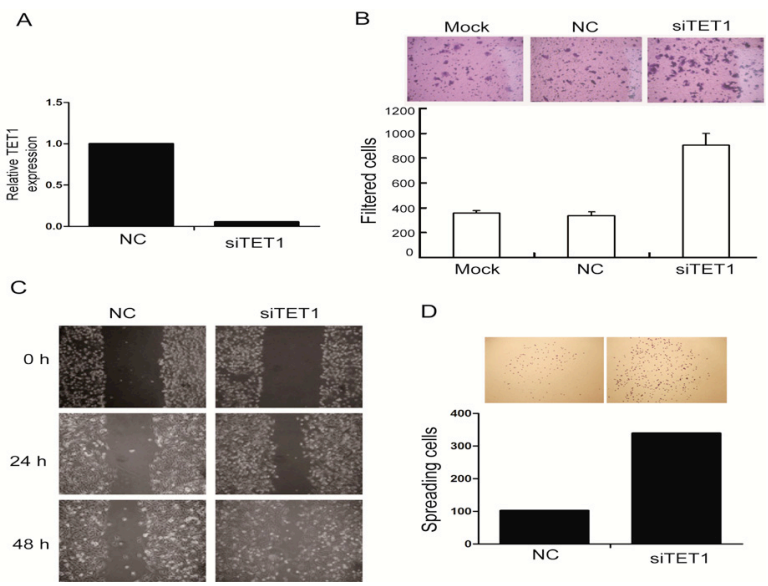

$\mathrm{D}$

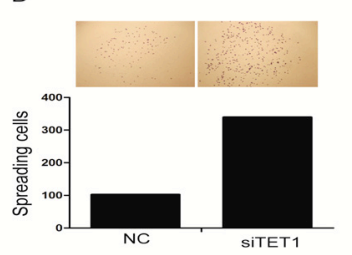

Figure 2. Knockdown of TET1 Strongly Promotes the Cell Migration, Invasion of Breast Cancer in vitro. A.The knockdown efficacy of TET1 by siRNA in BT474 cells. The indicated stable cells were transfected with siTET1 or negative control siRNA (NC) as indicated for $48 \mathrm{hrs}$, and the TET1 mRNA level was measured by qRT-PCR. B. Invasive abilities of the indicated stable cell lines in vitro was measured by transwell assay as described in "Materials and methods". Bars correspond to mean+ standard error. C. The cell ability of wound gap closure was dramatically enhanced by knocking down TET1. D. The cell ability of spreading was dramatically enhanced by knocking down TET1 control as determined in Figure 2A. Cells spreading on matrigel was then assessed for transfected cells , Figure 2D shows that siRNA TET1 promoted cell spreading.

\section{TET1 was down-regulated in breast cancer tissues with} lymph node metastasis

Given that the mRNA level of TET1 owned major differences between breast cancer cells with different metastatic capacity, we then detected its mRNA level in breast cancer clinical samples. TET1 mRNA levels in 28 pairs of freshly frozen primary breast cancer tissue and breast cancer with lymph node metastasis tissues were evaluated using qRT-PCR. As expect, the mRNA level of TET1 was significantly down-regulated in breast cancer with lymph node metastasis compared to primary breast cancer tissues (Figure 3).

Promoter methylation level of TET1 was correlated with metastasis status of patients with breast cancer

To examine whether the reduced TET 1 mRNA level was linked to the hypermethylation of TET1 promoter, we analyzed the methylation level of 56 breast cancer samples including 28 primary breast cancer tissues and 28 breast

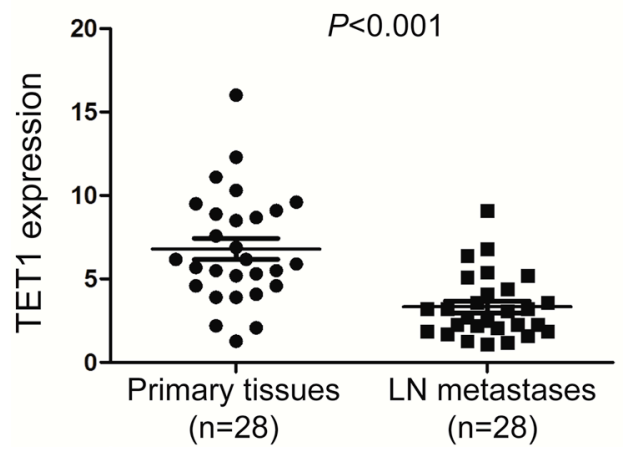

Figure 3. The mRNA Levels of TET1 were Lower in the Metastatic Breast Tumor Tissues in Lymph Node than the Breast Primary Tissues. The statistical results of qRT-PCR for the breast cancer primary tissues and their paired LN metastases using a paired t test $(\mathrm{P}<0.001)$. The dots represent the scores, while the bars indicate the $\mathrm{SD}$. $n=28$. Primary tissues: primary breast cancer tissues, $\mathrm{LN}$ metastases: the metastatic tumor tissues in lymph node

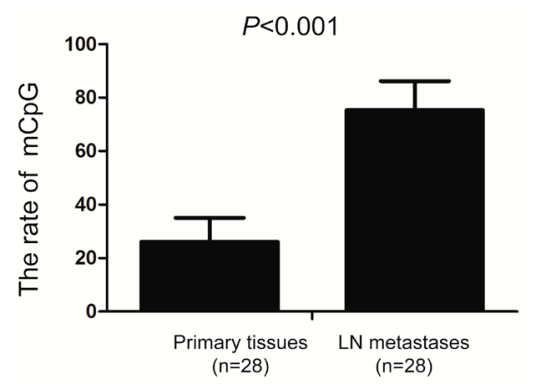

Figure 4. The Methylated Rates of TET1 Promoter were Higher in the Metastatic Breast Tumor Tissues in Lmph node than the Breast Primary Tissues Using a Paired t test $(\mathbf{P}<\mathbf{0 . 0 0 1})$. The methylation rates of $\mathrm{CpG}$ sites in the TET1 promoter were measured by BSP, as described in the materials and methods section. Primary tissues: primary breast cancer tissues, $\mathrm{n}=28$. LN metastases: the metastatic tumor tissues in lymph node., $\mathrm{n}=28$. The bars indicate the SD 
cancer tissues with lymph node metastasis using BSP. The mean TET1 methylation level is significantly higher in breast cancer tissues with lymph node metastasis than primary breast cancer tissues (Figure 4). These results indicated that the promoter hypermethylaiton of TET1 may be one of the mechanisms for the down-regualtion of TET1 in highly metastatic cells of breast cancer.

\section{Discussion}

Breast cancer metastasis is a major cause for cancerrelated death in women(Xu et al., 2014), breast cancer metastasis involves the spreading of cancer cells from the primary site to distant sites included lymph node, lungs, liver, bone, and brain. However, the etiology of breast cancer metastasis is unclear and its diagnosis markers are also rare.

TET1 protein was identified as a tumor suppressor gene in most of cancers included breast cancer. Here, our results showed that the expression of TET1 decreased in high metastatic ability of breast cancer cell lines and patient tissues, which may be as a valuable diagnosis for the breast cancer patients with metastasis. Furthermore, depletion of TET 1 resulted in enhanced migratory ability of BT474 cells, which is consistent with that observed in a previous study using other methods to detect cell migratory ability. However, in this study we paid particular attention to the reason why TET1 expression downregulated in high metastatic ability of breast cancer cells. It was reported that TET1 can bind and demethylate its own promoter in breast cancer cells, and enhancing its own expression(Sun et al., 2013). Our data also revealed that the hypermethylation of TET1 promoter in high metastatic breast cancer cells maybe result in its low expression.

In conclusion, our data indicated that TET1 expression was reduced and was inversely correlated with its promoter methylation level in high metastatic breast cancer cells. These findings provide a theoretical foundation for the possibility of TET 1 expression and its promoter hypermethylation as a potential diagnosis candidate for breast cancer metastasis. In order to explore this possibility, further studies on more detailed reason why there is hypermethylation on its promoter are required.

\section{References}

Cao J, Yang X, Li WT, et al (2014). Silencing of COX-2 by RNAi modulates epithelial-mesenchymal transition in breast cancer cells partially dependent on the PGE2 cascade. Asian Pac J Cancer Prev, 15, 9967-72.

Ciccarone F, Valentini E, Bacalini MG, et al (2014). Poly(ADPribosyl)ation is involved in the epigenetic control of TET1 gene transcription. Oncotarget.

Gan H, Wen L, Liao S, et al (2013). Dynamics of 5 -hydroxymethylcytosine during mouse spermatogenesis. Nat Commun, 4, 1995.

Gong C, Qu S, Lv XB, et al (2014). BRMS1L suppresses breast cancer metastasis by inducing epigenetic silence of FZD10. Nat Commun, 5, 5406.

$\mathrm{He}$ YF, Li BZ, Li Z, et al (2011). Tet-mediated formation of 5-carboxylcytosine and its excision by TDG in mammalian DNA. Science, 333, 1303-7.
Hong R, Shen MH, Xie XH, et al (2012). Inhibition of breast cancer metastasis via PITPNM3 by pachymic acid. Asian Pac J Cancer Prev, 13, 1877-80.

Hsu CH, Peng KL, Kang ML, et al (2012). TET1 suppresses cancer invasion by activating the tissue inhibitors of metalloproteinases. Cell Rep, 2, 568-79.

Kudo Y, Tateishi K, Yamamoto K, et al (2012). Loss of 5-hydroxymethylcytosine is accompanied with malignant cellular transformation. Cancer Sci, 103, 670-6.

Li J, Qiu DM, Chen SH, et al (2014). Suppression of human breast cancer cell metastasis by coptisine in vitro. Asian Pac J Cancer Prev, 15, 5747-51.

Lian CG, Xu Y, Ceol C, et al (2012). Loss of 5-hydroxymethylcytosine is an epigenetic hallmark of melanoma. Cell, 150, 1135-46.

Liu Y, Li H, Feng J, et al (2013). Lin28 induces epithelial-tomesenchymal transition and stemness via downregulation of let-7a in breast cancer cells. PLoS One, 8, 83083.

Lv XB, Jiao Y, Qing Y, et al (2011). miR-124 suppresses multiple steps of breast cancer metastasis by targeting a cohort of pro-metastatic genes in vitro. Chin J Cancer, 30, 821-30.

Mariani CJ, Vasanthakumar A, Madzo J, et al (2014). TET1mediated hydroxymethylation facilitates hypoxic gene induction in neuroblastoma. Cell Rep , 7, 1343-52.

Mercher T, Quivoron C, Couronne L, et al (2012). TET2, a tumor suppressor in hematological disorders. Biochim Biophys Acta, 1825, 173-7.

Pastor WA, Aravind L, Rao A (2013). TETonic shift: biological roles of TET proteins in DNA demethylation and transcription. Nat Rev Mol Cell Biol, 14, 341-56.

Sun M, Song CX, Huang H, et al (2013). HMGA2/TET1/ HOXA9 signaling pathway regulates breast cancer growth and metastasis. Proc Natl Acad Sci U S A, 110, 9920-5.

Tahiliani M, Koh KP, Shen Y, et al (2009). Conversion of 5-methylcytosine to 5-hydroxymethylcytosine in mammalian DNA by MLL partner TET1. Science, 324, 930-5.

Truong PK, Lao TD, Doan TP, et al (2014). BRCA1 promoter hypermethylation signature for early detection of breast cancer in the Vietnamese population. Asian Pac J Cancer Prev, 15, 9607-10.

Wu H, Zhang Y (2014). Reversing DNA methylation: mechanisms, genomics, and biological functions. Cell, 156, 45-68.

Xu Y, Gao XD, Lee JH, et al (2014). Cell type-restricted activity of hnRNPM promotes breast cancer metastasis via regulating alternative splicing. Genes Dev, 28, 1191-203.

Yang H, Liu Y, Bai F, et al (2013). Tumor development is associated with decrease of TET gene expression and 5-methylcytosine hydroxylation. Oncogene, 32, 663-9. 